\title{
BETA PICTORIS AND OTHER SOLAR SYSTEMS
}

\author{
PAWEL ARTYMOWICZ \\ Stockholm Observatory, Stockholm University, S-133 36 Saltsjöbaden, Sweden
}

Received: 3 December 1999; Accepted: 16 December 1999

\begin{abstract}
Planetary systems come in a bewildering variety of shapes and sizes. In addition to the exoplanetary systems with giant planets, found in surveys of stellar radial velocity variations, an overlapping class of dusty disk-containing solar systems exists. The disks include large quantities of meteoroids and dust, and a varying complement of gas. Their solid material represents 'replenished' dust born in the collisions/sublimation of planetesimals perturbed by planets. We present several such systems, including HR 4796A, HD 141569, HD 100546, and the prototypical replenished disk of Beta Pictoris. We discuss the composition, physical processing, and migration of dust in the disks, their evolutionary status, and the evidence of embedded planets.
\end{abstract}

\section{Diversity of Solar Systems}

In 1992, three Earth-class planets orbiting around pulsar PSR 1257+12 were serendipitously found (Wolszczan and Frail, 1992). This, and a later discovery by Mayor and Queloz (1995) of a Jupiter-mass companion to the star 51 Pegasi at a distance of only $a=0.05 \mathrm{AU}$, touched off an avalanche of discoveries by Doppler technique (Marcy and Butler, 1998) that in 1995-1999 led to as many as 29 convincing exoplanet candidates ( $c f$. Schneider, 1999). With the discovery of the transits of a giant gaseous planet of mean density equal to $0.4 \mathrm{~g} / \mathrm{cm}^{3}$ in HD 209458 (Charbonneau et al., 2000), any lingering doubt about the nature of the companions disappeared.

The ancient debate has thus been settled, concerning the unique $v s$. non-unique status of our Solar System. Still, we are not sure how common are the planetary systems of our type, with giant and terrestrial planets on low-eccentricity orbits. Surprisingly, most exoplanets found to date, due to a poorly known combination of observational selection and true statistics, do not resemble the Solar System counterparts. Their orbits are most often too close to their stars despite the Jupiter-type masses of these bodies, in sharp contrast with the terrestrial planets in the inner Solar System, or too elongated. Chemical composition of host stars appears to be correlated with the presence of giant exoplanets on tight (and only such) orbits, but there is so far no satisfactory explanation why this might be so. Ironically, PSR $1257+12$ remains the only truly solar-like system as regards the orbits and masses of the planets, despite the fact that very few millisecond pulsars have planets, let alone habitable ones.

Are exoplanetary systems built according to a blueprint, which differs from the one worked out in the past decades if not centuries for our own system? Not necessarily so. For more than a decade, we have studied another type of planetary systems: dusty circumstellar disks around main-sequence stars. Contrary to the perplexing situation with radial velocity systems, dusty disk systems better match the standard expectations about the mineralogy, spatial extent etc. (maybe because we still know little about their planets).

Space Science Reviews 92: 69-86, 2000.

(C) 2000 Kluwer Academic Publishers. Printed in the Netherlands. 
Those disks descend from the primordial solar nebulae that accompany forming stars of intermediate and low masses (such as solar-type stars). Late evolutionary stages of disk systems, following the loss of the hydrogen+helium gas from disks, are observed as a class of infrared-emitting systems known as Vega-type, Vegaexcess or $\beta$ Pic-type systems. Detected by IRAS and ISO satellites thanks to the presence of a large area (up to $\sim 10^{30} \mathrm{~cm}^{2}$ ) of solid grains much different from the ISM dust, complemented by a varying but small amount of gas, the Vega-type systems comprise as many as $15 \%$ of nearby main-sequence field stars of type A$\mathrm{K}$ (for review see Lagrange et al., 2000). Such systems were quickly realized to represent a stage in planetary system evolution (e.g., Aumann et al., 1984; Smith and Terrile, 1984), although it was not immediately clear which one (some were suggesting a stage of "snowballing" necessary to build large solid particles). An opposite view, that solids in the disks are intensely eroded and reduced to dust, rather than accumulating, now prevails. Planetesimals, i.e. pristine comets and small asteroids, replenish the dust disks via ice sublimation and collisions. The disks' ages range typically from a few to a few tenths of a Gyr (Artymowicz, 1997; VidalMadjar et al., 1998; Lagrange et al., 2000). High-resolution imaging has revealed structure in some of the disks implying a possible influence of planetary masses.

Finally, an important finding by Dominik et al. (1998) of a Vega-type excess from $55 \rho^{1}$ Cancri, a star orbited by a hot Jupiter-type planet, provided the first relatively unambiguous link between radial velocity and Vega-type systems. The presence of the disk has been confirmed by imaging (Trilling and Brown, 1998), and two more dust disks in the radial velocity systems $\rho$ CrB and HD 210277 have been found (Trilling, private communication)

\section{HD 100546: Mineralogy of Solids and Possible Cometary Activity}

Cosmochemistry predicts that the solids precipitating in solar-composition disks have, broadly speaking, chondritic mineralogy. Most of $\mathrm{C}$ and $\mathrm{O}$ bind into gaseous $\mathrm{CO}$, while the 'unused' O captures the abundant $\mathrm{Si}, \mathrm{Mg}$, and $\mathrm{Fe}$ (as well as less abundant refractory elements) in silicate grains at temperature $T<1400-1700 \mathrm{~K}$. Such temperatures were reached in the aftermath of gas cloud collapse near the protostar only. Forsterite $\mathrm{Mg}_{2} \mathrm{SiO}_{4}$ (magnesium olivine) and enstatite $\mathrm{MgSiO}_{3}$ (a pyroxene) are the most important abundant silicate minerals above $T \sim 500 \mathrm{~K}$ (Wood and Hashimoto, 1993). Ca, Al, and Na-containing diopside and albite contribute $<20 \%$ of solids. Below $500 \mathrm{~K}$ (unless kinetically prohibited) $\mathrm{Fe}$, first condensed in separate $\mathrm{Fe}$ and $\mathrm{FeS}$ grains, oxidizes and becomes incorporated into the silicate solid solution of olivines $(\mathrm{Mg}, \mathrm{Fe})_{2} \mathrm{SiO}_{4}$, and pyroxenes $(\mathrm{Mg}, \mathrm{Fe}) \mathrm{SiO}_{3}$, with a typical $\mathrm{Mg}$ :( $\left.\mathrm{Fe}+\mathrm{Mg}\right)$ number from 5:1 to 1:1. In the cold outer part of the nebula interstellar silicates and ices may be preserved $\left(\sim 90 \% \mathrm{H}_{2} \mathrm{O}\right.$, up to $4 \% \mathrm{CO}$, and some trace species like $\mathrm{CO}_{2}$, $\mathrm{NH}_{3}, \mathrm{CH}_{4}, \mathrm{~N}_{2}$ ice) and incorporated in comets at $r \gtrsim 20 \mathrm{AU}$. But only $1 / 3$ to $1 / 2$ of the mass of an "icy" body formed in the standard nebula (comets, Pluto/Charon, some outer Solar System satellites) is ice ( $c f$. also Prinn, 1993). 


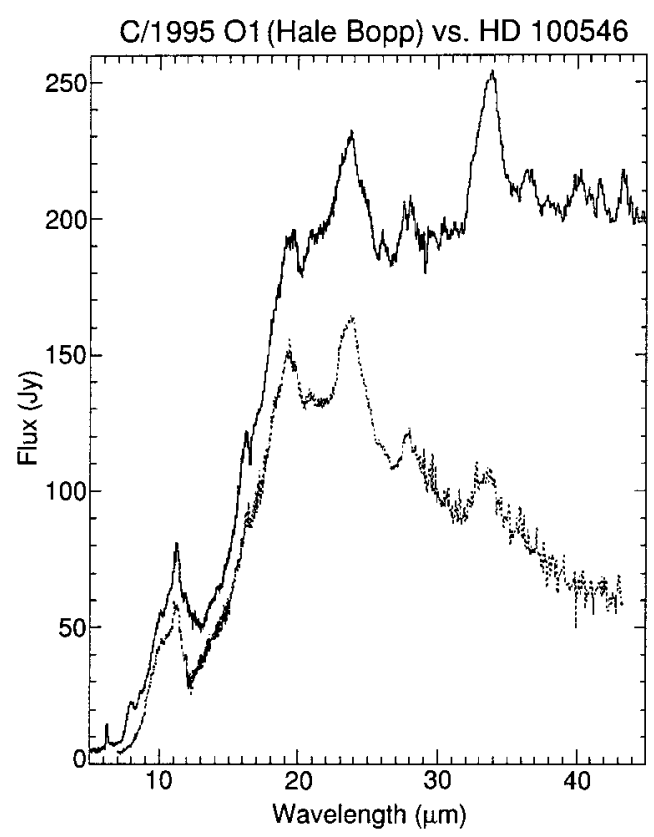

Figure 1. The comparison of ISO spectra of the dust in the circumstellar disk of a young star HD 100546 (upper curve), and comet Hale-Bopp dust (lower curve). Emission features of crystalline forsterite, amorphous olivine, $\mathrm{FeO}$, and $\mathrm{PAH}$ molecules have been identified in the disk spectrum (from Malfait et al., 1998).

To confront these expectations with astronomical reality, we discuss here the recent findings about HD 100546, a member of the Herbig Ae/Be class of PMS (pre-main sequence) stars which are somewhat more massive and luminous than the Sun, but otherwise 'solar-like'. Its similarity with other disk systems has been strengthened recently by the thermal radiation imaging of a disk-like structure at 10 and $20 \mu \mathrm{m}$ by Pantin and Lagage (private communication). The system is $\sim 10 \mathrm{Myr}$ old, and therefore belongs to the transitional IR-excess systems that may or may not have the primordial gas in the disk. Malfait et al. $(1998 ; 1999)$ obtained the SWS and LWS ISO spectra of the system. A variety of emission features of carbon- and oxygen-rich dust were found, most notably from crystalline forsterite. In contrast, in the interstellar medium and HII regions the silicate dust appears to be mostly amorphous. The crystallinity of dust in an extended, cold disk $(T<250 \mathrm{~K})$ is therefore quite intriguing. Have the amorphous minerals been annealed at moderate $T \approx 900 \mathrm{~K}$, or recrystallized after more extreme heating? While we have no definite answers yet (global mixing or circulation in the nebula may be required), we note that this problem concerns in equal degree the partial crystallinity of comets in the solar system. In fact, as Malfait et al. (1998; 1999) clearly show, in young planetary systems we are seeing dust very similar to the cometary dust. The lower graph in Fig. 1 gives the SWS/ISO spectrum of comet Hale-Bopp. Some IDPs (interplanetary dust particles) have similar emissivity curves. This resemblance, which was first noted in $\beta$ Pic, now extends to many related systems (Sitko et al., 1999).

Recently, Wooden et al. (1999) determined that comet Hale-Bopp's dust likely contains both the amorphous and the crystalline silicates (olivines, pyroxenes). Interestingly, the inferred fraction of $\mathrm{Mg}$-rich $[\mathrm{Mg}:(\mathrm{Mg}+\mathrm{Fe}) \approx 0.9]$ crystalline py- 
roxene in the dust is as high as $90 \%$ but its $10 \mu \mathrm{m}$-band emission features are only seen near the periastron passage, because such Mg-rich pyroxenes are more transparent than other types of silicates and have much lower $T(r)$ values.

Spectral resemblence to comets is not a sufficient proof that comets, as we know them, are present in a given other system. However, in HD 100546 there is some independent supporting evidence. HD 100546 may contain a huge swarm of comets making close passages and transits in front of the star, causing absorption line variability (episodic redshifts) observed in that system (Grady et al., 1997). Again, variability itself is not a sufficient proof of comets. Lagrange et al. (2000) are critical toward a recent trend to invoke comets for explanation of all forms of line variability. They claim that since a large preponderance of redshifted transient events is seen at medium to low velocities in many systems, the evaporating comets cannot be the general explanation for the spectroscopic phenomena. Such comets would cause a large number of blueshifted events as well. In case of HD 100546, however, we are faced with two indirect lines of evidence and should not dismiss the comets lightly.

The ISO spectrum of another object, HD 142527, differs markedly from that of HD 100546 and other objects in a similar evolutionary stage and with a similar broad-band energy distribution. Malfait et al. (1999) find no correlation between the spectral dust signatures (including crystallinity) and the stellar parameters such as age. More work is needed to clarify this important evolutionary issue and crystallize the theoretical thought.

\section{The Narrow Disk of HR 4796A}

HR 4796A is an important example of an "old PMS star" or alternatively a "young MS star" (Lagrange et al., 2000), evolved beyond the T Tau/Herbig AeBe stage but with denser dust disks than the Vega/ $\beta$ Pic prototypes, possibly including a significant if not primordial gas component. This A0V star has the highest IR disk fractional dust luminosity or "dustiness factor", $f_{\mathrm{d}}=L_{\text {dust }} / L_{*}=5 \times 10^{-3}$, among MS A-type stars. For comparison, $\beta$ Pic has a two times smaller $f_{\mathrm{d}}$. Spectroscopy of the M-type companion (HR 4796B, proj. distance $500 \mathrm{AU}$ ) yields an 8-10 Myr age for the system (e. g., Jura et al., 1998).

Several groups (Koerner et al., 1998; Jayawardhana et al., 1998; Schneider et al., 1999) recently imaged the dust disk, first in the $18 \mu \mathrm{m}$ thermal radiation band, and later in the scattered near-IR light by NICMOS/HST. A disk inclined 15-20 away from edge-on was found (Fig. 2, right-hand panel; Schneider et al., 1999). Impressive agreement with IR imaging was obtained with a simple power-law model of dust distribution, confined to radii from about 55-120 AU from the star (Koerner et al., 1998). The main disk seems narrow as a ring, quite unlike the similarly dusty $\beta$ Pic disk which extends to a projected radius of $>1200 \mathrm{AU}$. This may be due to the dynamical truncation of the disk by HR 4796B, or sensitivity limitations.

Telesco et al. (2000) re-observed the system at $10 \mu \mathrm{m}$ and compared the 10 and $20 \mu \mathrm{m}$ images, from which they obtained a (model-dependent) conclusion that 


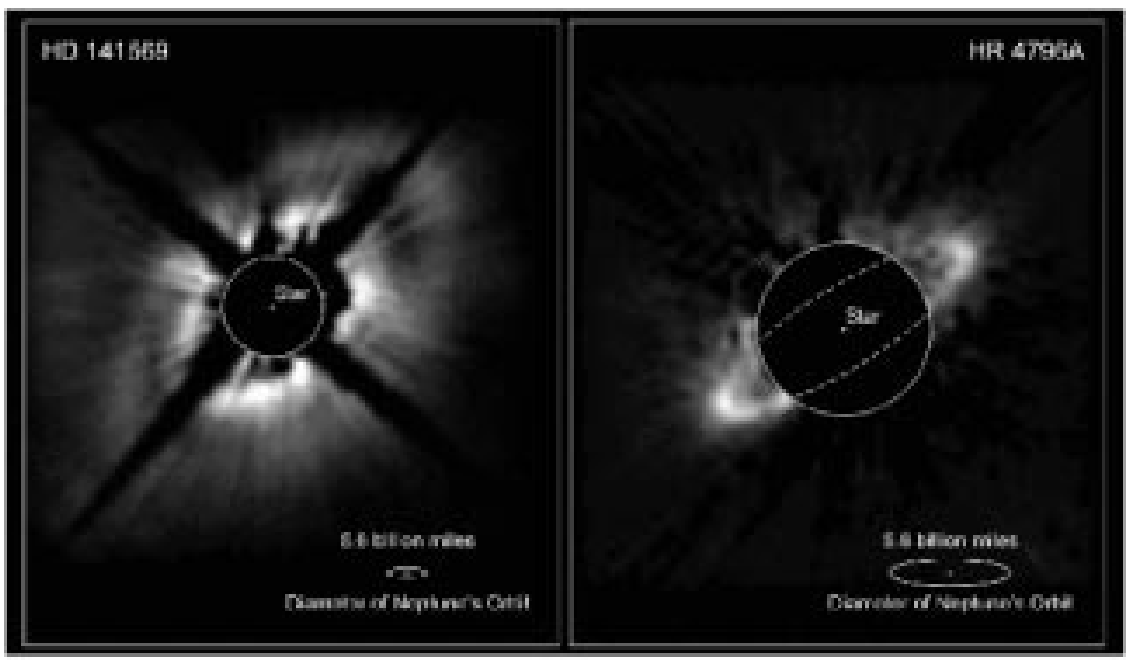

Figure 2. The dust disks around HD 141569 (left panel) and HR 4796A (right panel), in the recent NICMOS/HST observations (Weinberger et al., 1999; Schneider et al., 1999). Is their structure due to embedded planets or dust migration?

the grains in the disk have a 2 to $3 \mu \mathrm{m}$ radius. This raised their immediate concern since such grains composed of the ever popular "astronomical silicate" material (a material not proven to existent that fits the ISM extinction curves) would have radiation pressure coefficient $\beta \approx 1.7$ and thus would actually be seen on escape trajectories from the system (Wyatt et al., 2000). This prompts us to discuss below the role of radiation pressure in early planetary systems.

\section{The Roles of Radiation Pressure in Dust Disks}

\subsection{Interactions of Planetary Disks With Interstellar Dust}

Are internal or external factors controlling the evolution of Vega-type systems? Are dusty disks a matter of their nature or nurture in (a hostile) ISM? Lissauer and Griffith (1989) proposed that sandblasting by ISM dust during rapid passages through atomic clouds depletes circumstellar disks; $\beta$ Pic which happens to have a small velocity with respect to the clouds would be spared the sandblasting. Whitmire et al. (1992) proposed that all the nearby Vega-type stars have recently passed through molecular cloud and were sandblasted, which generated the finer dust from meteoroids. Artymowicz and Clampin (1997) revisited the issue and found that the ISM neither creates nor destroys the disks around A-type stars, mainly because the ISM grains are repelled by radiation pressure from several to a few dozen times stronger than stellar gravity; they argued that the 'internal sandblasting' normally dominates over ISM sandblasting out to radii of order $400 \mathrm{AU}$, at which the ISM may cause slight disk asymmetries. 


\subsection{Size Distribution and Kinematics of Radiation-AfFected Dust}

Radiation pressure equalling one-half the gravity $(\beta=0.5)$ of the star suffices for the escape to infinity of small grains (released from parent bodies on circular orbits). The dust below the corresponding radius $s_{\beta}$ will thus escape. Peak values of $\beta$ much larger than unity are actually found around the known Vega stars, reaching several dozen in the case of Vega itself. Much of the detailed work was restricted initially to $\beta$ Pic ( $c f$. Artymowicz, 1997). However, the effects of radiation are quite similar in different systems (e.g., Artymowicz and Clampin, 1997). For instance, the luminosity differences between the stars will simply shift the "blow-out grain radius' but will not change the characteristic shape of the grain size distribution, which must have a knee or cut-off around that radius.

The escaping dust fraction in the solar system is called $\beta$-meteoroids, as opposed to larger particles on bound orbits, which are sometimes called $\alpha$-meteoroids. Normally, it is expected that only $\alpha$-meteoroids will be observed due to their much longer residence time. Only if the disk is dense and collisions frequent enough, the combined area of escaping collisional debris could become larger than that of $\alpha$-meteoroids, as in the particle size distribution of HR 4796A (Telesco et al., 2000; Wyatt et al., 2000). But it is unclear if the disk is dense enough.

\section{Collisional Dynamics and Avalanches of Dust in Disks}

Our preliminary calculations show the possibility of the so-called dust avalanches (Artymowicz, 1996) in HR4796A and, in fact, any disk with similar dimensions and dustiness $\left(f_{\mathrm{d}}=5 \times 10^{-3}\right.$ in HR 4796A). The concept of an avalanche or, in other words, a chain reaction of outflowing debris is illustrated in Fig. 3. Three kinds of particles are distinguished in the figure. In addition to $\alpha$ and $\beta$ particles, we define the $\gamma$-meteoroids as such (very small) grains, whose dynamics are strongly affected by gas drag. Slowed down in their outflow by the drag force, $\gamma$ meteoroids do not contribute significantly to dust processing, and we ignore them for a moment ( $c f$., however, the next subsection). Mutual collisions of stable disk particles $(\alpha+\alpha)$ and the bombardment of disk grains by outflowing $\beta$-meteoroids $(\alpha+\beta)$ contribute to the creation of fresh $\beta$-meteoroids, subsequently accelerated by radiation pressure force. On the average, $N+M$ fresh $\beta$-meteoroids per collision are produced ( $N$ from cratering/erosion and $M$ from catastrophic disruption). Since the probability for a given $\beta$ particle to strike a disk grain while crossing the disk annulus with optical thickness $d \tau$ is roughly equal to $d \tau$, a toy model of the growth of an avalanche can be constructed by neglecting, among others, the need for a grain to accelerate over a finite radial distance before collisions can become strongly erosive/disruptive. The growth equation for the number $n$ of particles in the avalanche has the form $d n=(N+M) n d \tau$. The solutions of the toy model are thus exponentially growing avalanches of the form $n(\tau)=n_{0} \exp \left[\int_{0}^{\tau}(N+M) d \tau\right]=$ $\exp [\tau(N+M)]$, with $n_{0}$ an arbitrary constant. The optical thickness of the disk along 


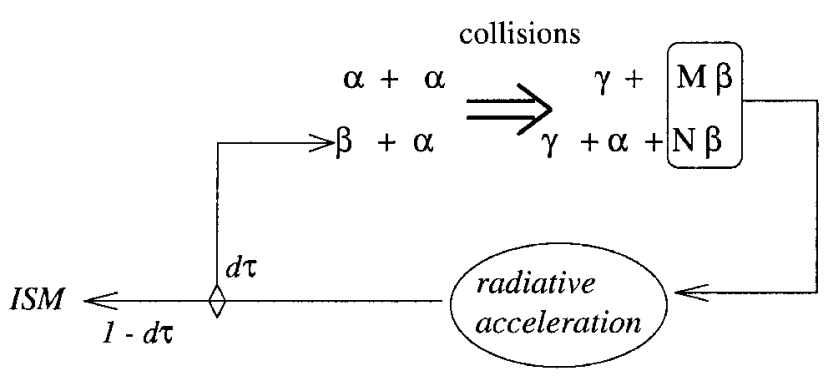

Figure 3. Dust avalanche concept. Collisions of stable $(\alpha)$ and outflowing $(\beta)$ meteoroids create fresh $\beta$-meteoroids, to be propelled by stellar radiation pressure. The positive feedback for an exponential growth of the avalanche is given by the optical thickness of a disk annulus, $d \tau$.

its midplane, $\tau$, is approximately its dustiness factor $f_{\mathrm{d}}$ divided by the flattening ratio $z / r \sim 0.1$. Therefore, a very large amplification factor of an avalanche of order exp $30 \approx 10^{13}$ may be obtained if $(N+M) f_{\mathrm{d}} \sim 30(z / r) \sim 3$.

From the physics of grain collision and more detailed models of a disk at radii $r \sim 100$ AU it follows that $N+M \sim 10^{2}-10^{3}$ (or $10^{2.5 \pm 0.5}$ ). Therefore, a plausible upper limit on the dustiness of gas-free disks exists in this model: $f_{\mathrm{d}}<3 /(M+N) \sim$ $10^{-2 \pm 0.5}$. This value is 4 times larger than that in $\beta$ Pic but only 2 times larger than that in HR 4796A. Hypothetical gas-free disks above that limit would tend to selfdestruct on exponentially short time scales unless and until they reduce their dustiness down to the limiting value. The details of the evolution are yet to be determined.

\subsection{INFERRING THE PRESENCE OF GAS}

The dust grinding rate which, in a steady-state collisional cascade, is also the mass loss rate from the largest parent bodies (planetesimals) can be evaluated in detailed disk modeling. Dividing the expected mass of the rocks in a typical protoplanetary disk (e.g., $\sim 120 \mathrm{M}_{\oplus}$, Artymowicz, 1997) by that rate yields the estimated lifetime of the whole disk (as opposed to the lifetime of the currently observed dust, which may be a thousand times shorter). Clearly, the disk's half-life should be longer than its age. This is not the case in HR 4796A, however. We obtained a preliminary estimate of 3 Myr disk self-destruction time. HR 4796A and its disk are $8 \pm 3$ Myr old.

This paradox can be resolved if we relax one or more of the model assumptions. The most natural solution is assuming an unobserved gas component with total mass at least $\sim 4$ times exceeding the dust mass, i.e. a fraction of $\mathbf{M}_{\oplus}$. This mass would be much smaller than the upper limit of $7 \mathrm{M}_{\oplus}$ of gas, obtained from observations in molecular emission lines (Zuckerman et al., 1995). Notice that the easiest method of direct detection of gas, via its absorption lines, may not be feasible in HR 4796A because of the viewing geometry with line of sight inclined by at least $1 / 3 \mathrm{rad}$, an angle several times larger than the opening angle $z / r \sim 1 / 10$ of a disk. Theory can thus be a valuable tool for predicting the presence of a hard-to-see gas component.

\subsection{Vega-Type System Classes}

The theoretical limit on the area of dust in Vega-type disks should modify the statistics of the observed fractional IR luminosity $f_{\mathrm{d}}$. Artymowicz (1996) considered 
the pre-ISO statistics of the Vega stars and obtained a bimodal histogram of $f_{\mathrm{d}}$. While there were many more examples (per $\log f_{\mathrm{d}}$ bin) of disks with $f_{\mathrm{d}}<10^{-3}$ and $f_{\mathrm{d}}>10^{-2}$, the only two systems in the $10^{-3}-10^{-2}$ bin were $\beta$ Pic and HR 4796A. This provides a strong support for the division of Vega-type systems into "gas-poor" and "gas-rich" based on a very simple and readily available diagnostic $\left(f_{\mathrm{d}}\right)$. It would be very interesting to revisit the observed statistics with a fuller sample of disks and proper account of selection effects, if any*.

\subsection{GAS-RELATED EFFECTS IN DISKS}

The gas can have many observable consequences. For example in HR 4796A it could slow down the otherwise rapid escape of very small grains ( $\gamma$-meteoroids). By the virtue of their slowness, $\gamma$-meteoroids might contribute significantly to the total area and observability of such fine dust. More generally, most gas-rich systems should be able to retain observable fraction of very small and transiently heated grains (which influences their light reprocessing and spectra) and/or PAH (polycyclic aromatic hydrocarbons). A correlation of PAH/small grain features with $f_{\mathrm{d}}$ is, indeed, present but awaits full analysis and description. Gas will also have a moderating effect on the overall dust processing rate in disks. If gas dynamics is truly important there, the HR 4796A disk may be a very promising laboratory for the study of distinctive nonlinear spiral density waves and resonantly truncated disk edges, hopefully indicating the position and orbit of planet(s) (e.g., Lubow et al., 1999). In contrast to this situation, a gas-poor disk like $\beta$ Pic will not be able to maintain a sharp edge with or without planets. The dust will in general be trapped only temporarily at the outer Lindblad resonances in the circumbinary disk (binary=star+planet). A gradual gap, washed out by large velocity dispersion in disk, might then appear (Roques et al., 1993). Sharpness of profiles and disk features is a hallmark of the presence of gas.

The aerodynamics of solid bodies in the dense primordial nebulae has been worked out a long time ago and finds applications in every modern dust evolution model ( $c f$. Weidenschilling, 1977, and in this volume). The same has now to be considered in the case of transitional systems with moderate gas density and non-negligible stellar radiation pressure. We outline a few interesting effects here, deferring details to a forthcoming publication.

Dust can migrate in disks both toward and away from the star, sometimes quite rapidly. The usual direction is inward, for the following reasons. In the solar nebula, there is a partial cancellation of the stellar gravity by the radial gradient of gas pressure, giving rise to a small decrease of orbital speed with respect to the Keplerian value $v_{\mathrm{K}}=\sqrt{G M / r}$; we can denote it by $\eta=\Delta v / v_{\mathrm{K}} \approx 0.005$. This causes an inward migration of solids due to the headwind $w=v_{\mathrm{K}} \eta$ experienced by particles, and the associated loss of angular momentum at a speed proportional to

* Notice that there is no obvious selection against discovering systems with, say, $f_{\mathrm{d}}=0.03$, as opposed to $f=0.003$ in sky surveys done in the past. 
$w$. The proportionality constant for large particles increases with decreasing size, for they are then better coupled to the gas (have larger area to mass ratio) while still orbiting along weakly perturbed Keplerian ellipses or circles. On the other hand, very small dust grains are coupled so well via strong gas drag force that they are almost frozen into the gas and corotate with it (at a sub-Keplerian speed $v_{\mathrm{K}}-w$ ). This causes a small, size-independent net radial acceleration (gravity being slightly larger than centrifugal force), balanced by the radial drag component. In this regime, migration speed increases linearly with the particle size because of the linearly decreasing drag force. Large-scale migration of solids in turbulent gas disks was recently modeled by Stepinski and Valageas (1997). The maximum migration speed at intermediate particle sizes, for which the stopping time (velocity divided by gas drag deceleration) is equal to the dynamical time $\Omega^{-1}$. Migration can remove such dust from a solar nebula.

In optically thin disks dust is subject to the combined gas drag and radiation pressure forces, which modifies the migration speed to $\dot{r} \sim(\beta / 2-\eta) v_{\mathrm{K}}$, where $\beta<1 / 2$ is the radiation to gravity ratio, and the coefficient of proportionality is a function of grain stopping time. For example, a $25 \mu \mathrm{m}$ radius grain in HR 4796A disk has $\beta \approx 0.2$ and has an average circulation velocity $v_{\phi}=\sqrt{G M(1-\beta) / r} \approx$ $(1-\beta / 2) v_{K}$. The orbital motion of that grain is slower than that of the gas, thus the particle feels the push of a backwind, gains angular momentum, and spirals outwards. In some cases outflow speed may be independent of particle size (over a certain size range), because the dust mobility of small (well-coupled) grains increases, while the radiation pressure drops with the grain size. PoyntingRobertson drag can be included in the analysis but it is never dominant except in very low-density disks, $e . g$., in our solar system.

\subsection{HD 141569: Planets OR DUST MimicRY?}

HD 141569, a system shown in the left-hand side of Fig. 2 is a quiescent Herbigtype star (type B9.5 Ve) surrounded by a light-scattering and IR emitting disk (inclination $40^{\circ}$ off edge-on). HD 141569 has a double-peaked $\mathrm{H} \alpha$ emission indicating a rotating gas disk close to the star, as well as gaseous $\mathrm{CO}$ detected at radius of order $90 \mathrm{AU}$ (Zuckerman et al., 1995; Weinberger et al., 1999). Details of its structure were imaged for the first time with NICMOS/HST at $1.1 \mu \mathrm{m}$ (Weinberger et al., 1999) and at $1.6 \mu \mathrm{m}$ (Augereau et al., 1999). The crossed dark radial stripes are artifacts of the observation method, but the division of the disk into an inner main part and an outer ring is intrinsic to the object, although over-exagerrated by grayscale map. Analysis of the $1.1 \mu \mathrm{m}$ observations yielded the radial profile of the vertical optical thickness $\tau(r)$ times the unknown albedo, which we reproduce in Fig. 4. The profile has a moderate dip at $r=250 \mathrm{AU}$, interpreted by Weinberger $e t$ al. (1999) as a sign of a planet residing in the disk at that radius. If this feature is indeed due to a planet, its eccentricity must be small (which requires formation in situ), and a moderate mass (on the order of Neptune's mass; this follows from gap 


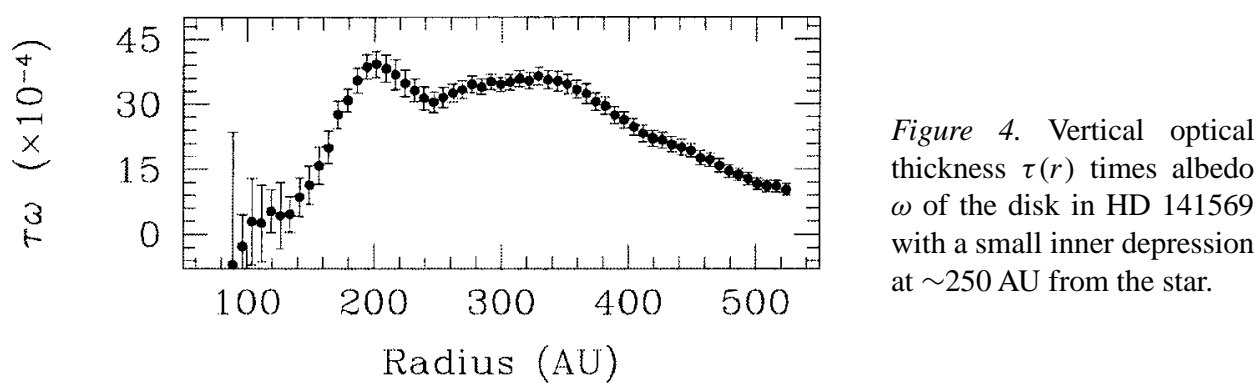

opening criteria). An obvious difficulty, however, arises in the formation theory because of the very large distance from the star. In the Solar System, the Kuiper belt region is 5 times closer and yet had insufficient mass (surface density) to form planets. Time needed to assemble planets at $250 \mathrm{AU}$ also strongly disfavors in situ formation. Outward migration to this large radius remains an unlikely possibility. In fact, the gap is not deep enough to stop a predicted rapid inward migration type I (Ward, 1997).

In this and similar cases one should avoid postulating planets indiscriminately to account for every morphological feature of the disk, unless supported by independent evidence. In HD 141569 other plausible explanations of the disk morphology exist, which naturally explain the puzzling differences in the appearence of the disk at the two wavelengths observed (Augereau et al., 1999, do not detect at $1.6 \mu \mathrm{m}$ the disk part inside $r=180 \mathrm{AU}$, prominent at $1.1 \mu \mathrm{m}$ ).

Firstly, if the disk is nearly optically thick in radial direction, shadowing of outer disk parts by inner disk parts in conjunction with a variable geometrical disk thickness might play a role, especially in the scattered light images like the NICMOS ones. At present we have no direct information on either vertical geometry or radial optical depth in this disk. Secondly, a variety of general scenarios can be constructed in which dust either originates or accumulates in a certain place in a disk, depending on dust grain size. We consider here the dust migration under the combined gas drag and radiation pressure forces.

As described above, the radial speed of particles changes with their size such that neither very small nor very large particles are able to migrate rapidly. Very large grains with $\beta<2 \eta \sim 0.01$ can be considered unaffected by radiation, and flow inward in a standard way. Such particles may transport, say, $0.1 \mathrm{~mm}$ and larger sand and stones toward the point in disk where the gas pressure reaches maximum, and the pressure support for gas rotation vanishes $(\eta=0$, no-headwind point). This point might either be near the stellar surface, in which case the dust evaporates before reaching the star, or somewhere near the inner edge of a disk tidally cut off by a planet or created in some other way. The accumulation of dust at maximum density point will eventually enhance dust collisions and the $\alpha$-meteoroids will be ground to fine dust. All meteoroids born in the collisions will migrate to the outer edge of a disk, $\beta$-meteoroids on nearly dynamical time scale (very fast), $\alpha$-grains moderately rapidly (in only $\sim 10$ orbital periods), and the $\gamma$-grains very slowly, so 


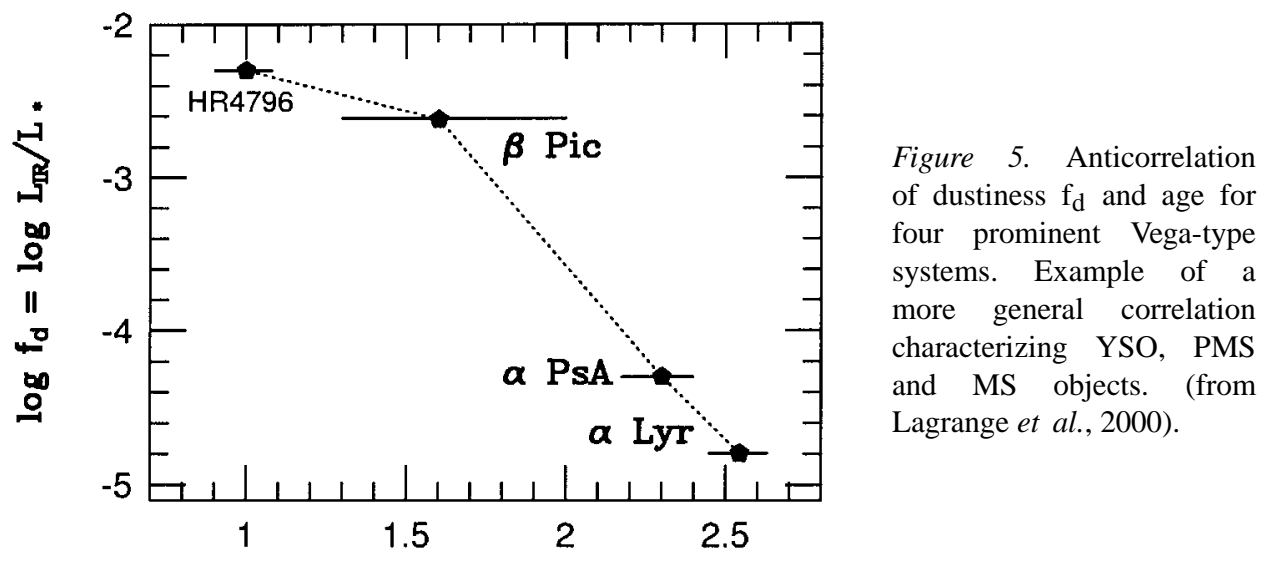

they alone will be visible enroute. At the egde, the $\gamma$-grains freed from the gas drag will escape, while $\alpha$-grains might be quasi-permanently trapped in orbits with eccentricity approximately equal to $e=\beta /(1-\beta)$, which assures that they are corotating with gas on the part of their elliptic orbit passing through dense gas (at pericenter). A qualitative picture is this: the inner disk seen at $1.1 \mu \mathrm{m}$ could consist of Rayleigh-scattering $\gamma$ particles (much less efficient at $1.6 \mu \mathrm{m}$ ), whereas the outer ring might consist of almost color-neutral scatterers. It could actually be brighter at the longer wavelength because of the material properties, such as the deep pyroxene absorption band around $0.9 \mu \mathrm{m}$. Quantitative models are yet to be constructed.

\subsection{Diminishing Dustiness of Solar Nebulae}

The first episode of loss of a large quantity of dust from the disk must coincide with the creation of the central clearing, of radius usually similar to the region occupied by Solar System planets. Such hallmark gaps or at least partial clearings are found without exception around the Vega-type stars, and are interpreted as a sign of the planetary influences. However, the presumably brief epoch of gap clearing has not been identified observationally yet.

The fact that at least $15 \%$ of nearby A, F, G, and K stars have far-IR dust excesses indicates that the disk live for sometimes more than 1 Gyr. Nevertheless, a general decrease of $\mathrm{f}_{\mathrm{d}}$ with age for PMS and MS objects is observed (e. g., Zuckerman and Becklin, 1993). Similar A-type stars HR 4796A, $\beta$ Pic, Fomalhaut, and Vega form a sequence of $f_{d}$ decreasing monotonically from ages of 10 to $350 \mathrm{Myr}$ (Fig. 5). Dominik et al. (1998) and Becklin et al. (1998) both found evidence in ISO surveys of nearby field stars that the fraction with detectable far-IR excesses drops from about $50 \%$ to about $15 \%$ after 500 Myr. Habing et al. (1999) noticed a sharp drop in that fraction at the age of $400 \mathrm{Myr}$, leaving only $9 \%$ of the older systems in their sample with detectable disks. 


\section{Beta Pictoris}

$\beta$ Pic is a thoroughly studied, although still far from fully explored Vega-type system (Backman and Paresce, 1993; Vidal-Madjar, 1994). A nearby and thus easily observed, edge-on disk around a seemingly normal* southern A5V-type star 1.7 times more massive than the Sun, $\beta$ Pic is now considered to be an analog of the early Solar System (Artymowicz, 1997; Vidal-Madjar et al., 1998; Lagrange et al., 2000).

\subsection{The Dust Component}

$\beta$ Pic has been imaged in $\mathrm{B}-\mathrm{I}, 12$ and $20 \mu \mathrm{m}$ bands, and sub-mm range. In addition, spectral information from UV to far IR was obtained. The total area of dust from visible light scattering is similar to that of Uranus' orbit filled tightly with dust. The geometrical thickness of the nearly edge-on disk is consistent with the theoretical expectations for a solar nebula $(z / r \approx 0.05)$, although extended wings at higher inclinations exist. Simultaneous modeling of thermal and scattered radiation was performed, using theoretical $T(r)$, temperature-radius relations, for specific assumed types of grains. Results show that $\beta$ Pic particles have a wide range of sizes, extending from submicron dust to at least mm-sized sand ( $c f$. Artymowicz, 1997). Most of disk surface area is contained in 1-20 $\mu \mathrm{m}$ particles causing, in conjunction with material properties, gray scattering of the visible and near-IR starlight. The disk has a $\sim 20 \mathrm{AU}$ radius central clearing which is not entirely empty. Warm silicates responsible for the 9-12 $\mu \mathrm{m}$ emission feature resembling closely comet Halley's spectrum (e.g., Knacke et al., 1993) and the thermal emission mapped by Lagage and Pantin (1994) reside in the clearing. They are dominated by particles with radius of order $2 \mu \mathrm{m}$.

New HST/STIS scattered-light images (Heap et al., 1999; Fig. 6) reach in to the projected distance $x \simeq 15 \mathrm{AU}$ from the star and constrain the vertical distribution of dust in the disk to have a sharp maximum at the disk midplane. The high spatialresolution images also show an inner 3-degree warp (or asymmetric bulge) in the mid-plane relative to that of the outer disk.

Good candidate materials for the main scattering disk include microscopically translucent silicates different than the ISM dust or anything else containing a large percentage of carbon or iron. Common, $\mathrm{Mg}$-rich olivines and pyroxenes already discussed in Sect. 3 (e.g., forsterite) stand out as an especially appealing possibility, not only because of the best models of $\beta$ Pic they yield but also because olivine and pyroxene are the most common rock-forming materials in the universe (consider, for example, the bulk of your home planet). An alternative material is a slightly darkened water ice, although some of its properties make it less relevant (Artymowicz, 1994, 1997).

The mean albedo $A>0.4$ is very unusual in comparison with surfaces of solar system bodies and most planetary rings; part of the reason may lie in the size

* Its currently accepted main sequence status, normal metallicity, and age of several dozen to several hundred Myr, withstood many challenges and claims in the past decade; $c f$. the cited literature. 


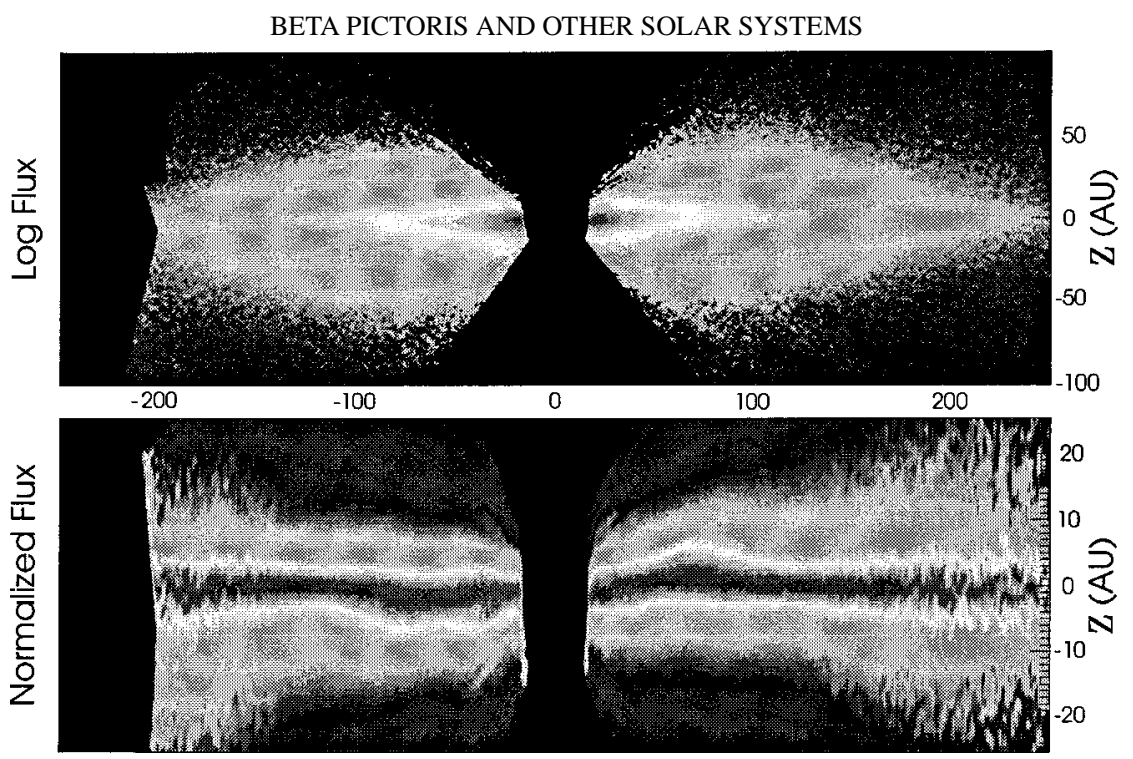

Figure 6. HST/STIS coronagraphic visible-light image of the $\beta$ Pic disk (Heap et al., 1999). The original lens-shaped image was stretched 4 times in the vertical direction and renormalized to the maximum flux in each CCD column, in order to emphasize the warp-like bulge extending to $>100 \mathrm{AU}$ on each side of the star.

distribution promoting efficient scattering, and part in the absence of chondritic abundances of carbon and iron/nickel. In any case, the typical $\beta$ Pic dust appears different than an unprocessed interstellar dust causing interstellar extinction ("astronomical silicate") both chemically and size-wise, which strengthens its ties with planetary materials.

Linear polarization of $\beta$ Pic disk, of order $15 \%$, can be reproduced using zodiacal light's scattering function and the $\beta$ Pic disk dust distribution. In common with the zodiacal light disk, $\beta$ Pic disk is undergoing a collisional evolution gradually destroying the large bodies and replenishing the dust. In our system, collisions are infrequent and Poynting-Robertson drag is the main process removing the dust. In contrast, in $\beta$ Pic collisions are very frequent due to the $10^{4}$ times larger optical depth of dust, limiting the lifetime of typical grains to dozens of orbital periods only. Most collisional products are blown out radiatively. In both systems thermal evaporation of icy particles and planetesimals probably occurs, but its contribution to the dust replenishment rate remains uncertain. In our system comets may plausibly contribute one third of zodiacal dust. In $\beta$ Pic it is very doubtful that evaporation of comets could replenish the massive and extended dust disk at the required rate of $\sim 1 M_{\oplus} / \mathrm{Myr}$, indicating collisions as the dominant erosional mechanism.

The gap size obtained from modeling of the MIR images is significantly smaller than the gap size obtrained from fits to the STIS visible image. This excludes a one-component model of dust, and requires that the warm (MIR emitting) particles be hotter and less symmetrically distributed in space than the colder dust responsible for scattering. If multi-temperature silicate dust conglomerate similar 
to that described by Wooden et al. (1999) in comet Hale-Bopp exists around $\beta$ Pic, the mixing ratio of minerals need not necessarily vary across the system. Detailed modeling has yet to be performed.

\subsection{The Gas and the Planetesimals}

The gaseous component of the disk produces narrow absorption lines (Ferlet and VidalMadjar, 1994; Lagrange et al., 2000). Two types can be distinguished.

Stable absorption lines: $\beta$ Pic (a 'shell star') exhibits narrow absorption lines of singly ionized metals superimposed on the rotationally broadened photospheric lines. These lines originate in some neutral (e.g., Na I, C I, CO) and many ionized species (Ca II, Mn II, Fe II, and Zn II). The basic result is that the column densities of these elements are all in the cosmic (solar) abundance ratios. This observed gas is not derived from a solar-abundance gas like that of the primordial solar nebula, however. The overall gas:dust mass ratio may be only $\leq 0.003$, or $<3 \times 10^{-5}$ times that in the solar nebula (Liseau and Artymowicz, 1998). Thus, the disk is almost gas-free. The likely source of gas are evaporating solid dust grains and macroscopic bodies.

Variable absorption lines: Indirect evidence for the conversion of solid bodies, with abundances and masses corresponding to $1-\mathrm{km}$ planetesimals, into hot plasma near the star was found in the time-variable absorption features (for review see Vidal-Madjar, 1994; Lagrange, 1995). The absorption events are almost always redshifted by up to $+300 \mathrm{~km} / \mathrm{s}$, about one-half of the free-fall velocity onto the star, but more commonly reach a ten times smaller infall velocity. The cometary/planetesimal near-grazing encounters may be responsible for the events, a scenario called Falling Evaporating Bodies or FEBs (Vidal-Madjar, 1994; Beust et al., 1994). Events occur up to 200 times per year, however much less than that some years. This, together with the large predominance of redshifted over blueshifted features, suggests that the FEBs belong to an orbital family, i. e. a group of bodies with similar orbits.

\subsection{Possible Planets Around $\beta$ Pic}

Being dozens of Myr old, the system seems mature enough to have planets, of which there are as yet no strong indirect indications. However, taken together the individual arguments discussed below make their existence likely.

The Observed Disk Thickness. The 0.1 radian half-opening angle of the disk, or in other words the dispersion of orbital inclinations of dust and its parent bodies, may require the presence of numerous $\left(10^{2}-10^{3}\right.$ ?) Moon-sized bodies in the disk.

FEBs approaching the star. Many researchers consider the existence of at least one planet as necessary for the very existence of FEBs (Levison et al., 1994; Beust and Morbidelli, 1996). Namely, the only force able to rearrange the orbital distances and eccentricities of initially nearly circular orbits of planetesimals (similar to orbits of most Kuiper disk objects) is the force of gravity of planets. Nothing short of planets is able to do it efficiently because, e. g., comet-sized planetesimals 
are only able to perturb their orbits to the relative speed (of order escape speed) measured in $\mathrm{m} / \mathrm{s}$, rather than the required $\mathrm{km} / \mathrm{s}$.

Photometric variability of the star. Lecavelier et al. (1995) analyzed archival photometric observations of $\beta$ Pic, and have noticed variations in the brightness of the star with no measurable color dependency. The most interesting of these was an unusual brightening by 0.04 mag within \pm 10 days from Nov 10,1981 . These variations were tentatively attributed to a planet or a giant comet cloud releasing forward-scattering dust (Lecavelier et al., 1995; Lamers et al., 1997).

The central clearing. The Kuiper disk has a gap with a relatively sharp edge at $r \sim 40 \mathrm{AU}$ caused by planetary perturbations of comets (its associated dust is still unobservable.) Calculations of Roques et al. (1993) show a similar effect a planet might have on the dust disk in $\beta$ Pic. This is an interesting possibility which should be studied further, including collisional physics.

Disk asymmetries. The disk is asymmetric in various ways (Kalas and Jewitt, 1995). Roques et al. (1993) and Liou and Zook (1999) have studied the nonaxisymmetric structures in disks subject to planetary perturbations, yielding possibly similar asymmetries. Their models cannot be directly applied to $\beta$ Pic because of differences between the Solar System physics used in the models (e.g., P-R effect), and the collisional-radiative nature of $\beta$ Pic disk. The origin of asymmetries is an open problem at present.

Disk warp. The warp or asymmetric bulging of the disk seen in Fig. 6 (lower panel) supports the planetary hypothesis. It is most easily explained by the influence of a planet or brown dwarf orbiting inside the masked region (Burrows et al., 1995; Mouillet et al., 1997). A planet on an inclined orbit acts on the planetesimal or meteoroid in the disk over secular time scales in such a way as if the planet (and the planetesimal) were rotating, mutually inclined, rings. Such rings behave like spinning tops: They precess at a rate proportional to the perturbing mass. The ascending node of the planetesimal orbit precesses with a period equal to its orbital period times $\sim\left(r / r_{\mathrm{p}}\right)^{2} M_{*} / M_{\mathrm{p}}$, where $M_{*}$ and $M_{\mathrm{p}}$ are the star and planet masses. The inner disk region at $r \sim 40 \mathrm{AU}$ could thus be effectively forced to wobble around the planet's, rather than disk's, plane of motion in just $15 \mathrm{Myr}$, if one Jupiter mass is present at $r=5.2 \mathrm{AU}$ like in the Solar System.

\section{Conclusions}

At the close of the millenium, we were fortunate to witness the discovery of both young and old planetary systems, $\beta$ Pic being the first young one to have been explored in some detail. We know that the extrasolar systems are not unique in the universe but fairly common, even if only 5 to $25 \%$ of all stars may harbor giant planets or easily resolved dust disks. Comparing the perplexing orbital diversity of exoplanets found in radial velocity surveys (Marcy and Butler, 1998; Schneider, 1999) with the findings on circumstellar disks described in the present paper, we must conclude that the former yielded more disparities between the observations 
and prior theoretical expectations. $\beta$ Pic-type systems provide a much closer analog to a stage in development of the Solar System then the exoplanets are an analog of the current Solar System, in the orbital sense. Planetary disks facilitate the study of the origin of both exoplanetary and solar systems, including details (such as mineralogy) difficult to obtain otherwise. Spectacular systems such as $\beta$ Pic are, of course, rare in the sky, because of their youth, which is understood to be brief and dirty (dusty), but may exemplify a common phase in the lives of most stars.

\section{Acknowledgements}

It is a pleasure to thank the ISSI staff for hosting an informative meeting, NFR (Swedish Nat. Sci. Board) for generous grants, and the editors of the present volume for their support and patience.

\section{References}

Artymowicz, P.: 1994, 'Modeling and Understanding the Dust Around Beta Pictoris', see Ferlet and Vidal-Madjar, 1994, pp. 47-65.

Artymowicz, P.: 1996, 'Vega-type Systems', in H. U. Käufl and R. Siebenmorgen (eds.), The Role of Dust in the Formation of Stars, Springer, Berlin, pp. 137-148.

Artymowicz, P.: 1997, 'Beta Pictoris: An Early Solar System?', Ann. Rev. Earth Planet. Sci. 25, 175-219.

Artymowicz, P., and Clampin, M.: 1997, 'Dust Around Main Sequence Stars: Nature or Nurture by the Interestellar Medium?', Astrophys. J. 490, 863-878.

Augereau, J. C., Lagrange, A. M., Mouillet, D., Monard, F.: 1999, 'HST/NICMOS2 Observations of the HD 141569 A Circumstellar Disk', Astron. Astrophys. 350, L51-L54;

Augereau, J. C., Lagrange, A. M., Mouillet, D., Papaloizou, J.C.B., Grorod, P. A.: 1999, 'On the HR 4796 A Circumstellar Disk', Astron. Astrophys. 348, 557-569.

Aumann, H. H., Beichman, C. A., Gillett, F. C., de Jong, T., Houck, J. R., Low, F. J., Neugebauer, G., Walker, R. G., Wesselius, P. R.: 1984, 'Discovery of a Shell Around Alpha Lyrae', Astrophys. J. 278, L23-L27.

Backman, D. E., and Paresce, F.: 1993, 'Main-sequence Stars With Circumstellar Solid Material: The Vega Phenomenon', in E. E. Levy, J. I. Lunine, and M. S. Matthews (eds.), Protostars and Planets III, Univ. Arizona Press, Tuscon, 1993, pp. 1253-1304.

Becklin, E. E., Silverstone, M., Chary, R., Hare, J., Zuckerman, B., Spangler, C., Sargent, A.: 1998, 'Dust Around Solar Mass Stars', Astrophys. Space Sci. 255, 113-118.

Beust, H., and Morbidelli, A.: 1996, 'Mean Motion Resonances as a Source for Infalling Comets Toward $\beta$ Pictoris', Icarus 120, 358-370.

Beust, H., Vidal-Madjar, A., Ferlet, R., and Lagrange-Henri, A.M.: 1994, 'Cometary-like Bodies in the Protoplanetary Disk Around $\beta$ Pictoris', Astrophys. Space Sci. 212, 147-157.

Burrows, C., Krist, J.E., Stapelfeldt, K. R., and the WFPC2 Investigation Definition Team: 1995, 'HST Observations of the Beta Pictoris Circumstellar Disk', Bull. Amer. Astr. Soc. 187, \#32.05 (abstract).

Charbonneau, D., Brown, T., Latham, D. W., and Mayor, M.: 2000, 'Detection of Planetary Transits Across a Sun-like Star', Astrophys. J. in press.

Dominik, C., Laureijs, R. J., Jourdain de Muizon, M., and Habing, H. J.: 1998, 'A Vega-like Disk Associated with the Planetary System of rho (1) Cnc', Astron. Astrophys. 329, L53-L56.

Ferlet, R. and Vidal-Madjar, A. (eds.): 1994, Circumstellar Dust Disks and Planet Formation, Gifsur-Yvette: Editions Frontières. 
Grady, C. A., Sitko, M. L., Bjorkman, K. S., Perez, M. R., Lynch, D. K., Russell, R. W., Hanner, M. S.: 1997, 'The Star-grazing Extrasolar Comets in the HD 100546 System', Astrophys. J. 483, 449456.

Habing, H., Dominik, C., Jourdain de Muizon, M., Kessler, M. F., Laureijs, R. J., Leech, K., Metcalfe, L., Salama, A., Siebenmorgen, R., Trams, N.: 1999, 'Disappearance of Stellar Debris Disks Around Main-sequence Stars After 400 Million Years', Nature 401, 456-458.

Heap, S. R., Linder, D. J., Lanz. T. M., Woodgate, B., Cornett, R., Hubeny, I., and Maran, S. P.: 1999, 'STIS Coronagraphic Observations of $\beta$ Pictoris', Astrophys. J., in press.

Jayawardhana, R., Fischer, S., Hartmann, L., Telesco, C., Pina, R., Fazio, G.: 1998, 'A Dust Disk Surrounding the Young A Star HR 4796A', Astrophys. J. 503, L79-L82.

Jura, M., Malkan, M., White, R., Telesco, C., Pina, R., Fisher, R. S.: 1998, 'A Protocometary Cloud Around HR 4796A?', Astrophys. J. 505, 897-902.

Kalas, P., and Jewitt, D.: 1995. 'Asymmetries in the Beta Pictoris Dust Disk', Astrophys. J. 110 794-804.

Knacke, R. F., Fajardo-Acosta, S. B, Telesco, C. M., Hackwell, J. A., Lynch, D. K., Russell, R. W.: 1993, 'The Silicates in the Disk of Beta Pictoris', Astrophys. J, 418, 440-450.

Koerner, D. W., Ressler, M. E., Werner, M. W., Backman, D.: 1998, 'Mid-Infrared Imaging of a Circumstellar Disk Around HR 4796: Mapping the Debris of Planetary Formation', Astrophys. J. 503, L83-L87.

Lagage, P. O., and Pantin, E.: 1994, 'Dust Depletion in the Inner Disk of Beta Pictoris as a Possible Indicator of Planets', Nature 369, 628-630.

Lagrange, A. M.: 1995, 'Observations of Disks Around Main Sequence Stars ( $\beta$ Pictoris)', Astrophys. Space Sci. 223, 19-43.

Lagrange, A. M., Backman, D., and Artymowicz, P.: 2000, 'Planetary Material Around Main Sequence Stars', in V. Mannings, A. Boss, S. Russell (eds.), Protostars and Planets IV, Univ. Arizona Press, Tuscon, 2000, in press.

Lamers, H. J., Lecavelier Des Etangs, A., and Vidal-Madjar, A.: 1997, 'Beta Pictoris Light Variations. II. Scattering by a Dust Cloud', Astron. Astrophys. 328, 321-330.

Lecavelier des Etangs, A., Deleuil, M., Vidal-Madjar, A., Ferlet, R., Nitschelm, C., Nicolet, B., Lagrange-Henri, A. M.: 1995, ' $\beta$ Pictoris: Evidence of Light Variations', Astron. Astrophys. 299, $557-562$.

Levison, H.F., Duncan, N. J., and Wetherill, G. W.: 1994, 'Secular Resonances and Cometary Orbits in the Beta Pictoris System', Nature 372, 441-443.

Liou, J.-C., and Zook, H. A.: 1999, 'Signatures of Giant Planets Imprinted on the Edgeworth-Kuiper Belt Dust Disk', Astrophys. J. 118, 580-590.

Liseau, R., and Artymowicz, P.: 1998, 'High Sensitivity Search for Molecular Gas in the Beta Pic Disk. On the Low Gas-to-dust Mass Ratio of the Circumstellar Disk Around Beta Pictoris', Astron. Astrophys. 334, 935-942.

Lissauer, J. J., and Griffith, C. A.: 1989, 'Erosion of Circumstellar Particle Disks by Interstellar Dust', Astrophys. J. 340, 468-471.

Lubow, S.H., Seibert, M., and Artymowicz, P.: 1999. 'Disk Accretion Onto High-Mass Planets', Astrophys. J., in press.

Malfait, K., Waelkens, C., Waters, L.B., Vandenbussche, B., Huygen, E., de Graauw, M. S.: 1998, 'The Spectrum of the Young Star HD 100546 Observed With the ISO', Astron. Astrophys. 332, L25-L28.

Malfait, K., Waelkens, C., Bouwman, J., de Koter, Waters, L.B.F.M.: 1999, 'The ISO Spectrum of the Young Star HD 142527', Astron. Astrophys. 345, 181-186.

Marcy, G. W., and Butler, R. P.: 1998, 'Detection of Extrasolar Giant Planets', Ann. Rev. Earth Planet. Sci. 36, 57-98.

Mayor, M., and Queloz, D.: 1995, 'A Jupiter-mass Companion to a Solar-type Star', Nature 378, 355-359. 
Mouillet, D., Larwood, J., Papaloizou, J., Lagrange, A. M.: 1997, 'A Planet on an Inclined Orbit as an Explanation of the Warp in the Beta Pictoris Disk', Monthly Not. Roy. Astron. Soc. 292, 896-904.

Prinn, R. G.: 1993, 'Chemistry and Evolution of Gaseous Circumstellar Disks', in E. E. Levy, J. I. Lunine, and M. S. Matthews (eds.), Protostars and Planets III, Univ. Arizona Press, Tuscon, 1993, pp. 1005-1028.

Roques, F., Scholl, H., Sicardy, B., Smith, B. A.: 1994, 'Is There a Planet Around $\beta$ Pictoris - Perturbations of a Planet on a Circumstellar Dust Disk. 1. The Numerical Model', Icarus 108, 37-58.

Schneider, J.: 1999, 'Extrasolar Planet Encyclopaedia Site', http://www.obspm.fr/planets

Schneider, G., Smith, B. A., Becklin, E., Koerner, D., et al.: 1999, 'NICMOS Imaging of the HR 4796A Circumstellar Disk', Astrophys. J. 513, 127.

Sitko, M., Grady, C. A., Lynch, D. K., Russell, R. W., Hanner, M. S.: 1999, 'Cometary Dust in the Debris Disks of HD 31648 and HD 163926: Two "Baby” $\beta$ Pic Systems', Astrophys. J. 510, 408-412.

Smith, B. A., and Terrile, R. J.: 1984, 'A Circumstellar Disk Around Beta Pictoris', Science 226, $1421-1424$.

Stepinski, T., and Valageas, P.: 1997, 'Global Evolution of Solid Matter in Turbulent Protoplanetary Disks. II. Development of Icy Planetesimals', Astron. Astrophys. 319, 1007-1019.

Telesco, C. M., Fisher, R.S., Pina, R. K., Knacke, R.F., et al.: 2000, 'Deep 10 and $18 \mu \mathrm{m}$ Imaging of the HR 4796A Circumstellar Disk: Transient Dust Particles and Tentative Evidence for a Brightness Asymmetry', Astrophys. J., in press.

Trilling, D., and Brown, R.H.: 1998, 'A Circumstellar Dust Disk Around a Star with a Known Planetary Companion', Nature 395, 775-777.

Vidal-Madjar, A.: 1994, ' $\beta$-Pic: The Gaseous Circumstellar Disk’, in Ferlet and Vidal-Madjar (1994), pp. 7-18.

Vidal-Madjar, A., Lecavelier des Etangs, A., and Ferlet, R.: 1998, ' $\beta$ Pic, a Young Planetary System?', Planet. Space Sci. 46, 629-648.

Ward, W. R.: 1997, 'Survival of Planetary Systems', Astrophys. J. 482, 211-215.

Weidenschilling, S.: 1977, 'Aerodynamics of Solid Bodies in the Solar Nebula', Month. Not. Roy. Astron. Soc. 180, 57-70.

Weinberger, A. J., Becklin, E.E., Schneider, G., Smith, B. A., Lowrance, P. J., Silverstone, M. D., Zuckerman, B., and Terrile, R.J.: 'The Circumstellar Disk of HD 141569 Imaged With NICMOS', Astrophys. J. 525, L53-L56.

Whitmire, D. P., Matese, J. J., Whitman, P. G.: 1992, 'Velocity Streaming of IRAS Main-sequence Disk Stars and the Episodic Enhancement of Particulate Disks by Interstellar Clouds', Astrophys. J. 388, 190-195.

Wolszczan, A., and Frail, D. A.: 1992, 'A Planetary System Around the Millisecond Pulsar PSR 1257+12', Nature 355, 145-147.

Wood, J., and Hashimoto, A.: 1993, 'Mineral Equilibrium in Fractionated Nebular Systems', Geochim. Cosmochim. Acta 57, 2377-2388.

Wooden, D. H., Harker, D.E., Woodward, C.E., Butner, H. M., Koike, C., Witteborn, F. C., McMurtry, Craig, W.: 1999, 'Silicate Mineralogy of the Dust in the Inner Coma of Comet C/1995 01 (Hale-Bopp) Pre- and Postperihelion', Astrophys. J. 517, 1034-1058.

Wyatt, M. C., Dermott, S. F., Telesco, C. M., Fisher, R. S., et al.: 2000, 'How Observations of Circumstellar Disk Asymmetries can Reveal Hidden Planets: Pericenter Glow and its Application to the HR 4796 Disk', Astrophys. J. in press.

Zuckerman, B., and Becklin, E.E.: 1993, 'Submillimeter Studies of Main-sequence Stars', Astrophys. J. 414, 793-802.

Zuckerman, B., Forveille, T., Kastner, J. H.: 1995, 'Inhibition of Giant-planet Formation by Rapid Gas Depletion Around Young Stars', Nature 373, 494-496.

Address for Offprints: Pawal Artymowicz, Stockholm Observatory, Stockholm University, S-133 36

Saltsjöbaden, Sweden; pawel@astro.su.se 